\title{
A CHARACTERIZATION OF MODULATION SPACES BY SYMPLECTIC ROTATIONS
}

\author{
ELENA CORDERO, MAURICE DE GOSSON, AND FABIO NICOLA
}

\begin{abstract}
This note contains a new characterization of modulation spaces $M^{p}\left(\mathbb{R}^{n}\right), 1 \leq p \leq \infty$, by symplectic rotations. Precisely, instead to measure the time-frequency content of a function by using translations and modulations of a fixed window as building blocks, we use translations and metaplectic operators corresponding to symplectic rotations. Technically, this amounts to replace, in the computation of the $M^{p}\left(\mathbb{R}^{n}\right)$-norm, the integral in the timefrequency plane with an integral on $\mathbb{R}^{n} \times U(2 n, \mathbb{R})$ with respect to a suitable measure, $U(2 n, \mathbb{R})$ being the group of symplectic rotations. More conceptually, we are considering a sort of polar coordinates in the time-frequency plane. In this new framework, the Gaussian invariance under symplectic rotations yields to choose Gaussians as suitable window functions. We also provide a similar characterization with the group $U(2 n, \mathbb{R})$ being reduced to the $n$-dimensional torus $\mathbb{T}^{n}$.
\end{abstract}

\section{INTRODUCTION}

The objective of this study is to find a new characterization of modulation spaces using symplectic rotations. Precisely, we are interested in those metaplectic operators $\widehat{S} \in M p(n, \mathbb{R})$, such that the corresponding projection $S:=\pi(\widehat{S})$ onto the symplectic group $S p(n, \mathbb{R})$ is a symplectic rotation. Let us recall that the symplectic group $S p(n, \mathbb{R})$ is the subgroup of $2 n \times 2 n$ invertible matrices $G L(2 n, \mathbb{R})$, defined by

$$
S p(n, \mathbb{R})=\left\{S \in G L(2 n, \mathbb{R}): S J S^{T}=J\right\},
$$

where $J$ is the orthogonal matrix

$$
J=\left(\begin{array}{cc}
0_{n} & I_{n} \\
-I_{n} & 0_{n}
\end{array}\right),
$$

$\left(I_{n}, 0_{n}\right.$ are the $n \times n$ identity matrix and null matrix, respectively). Here we consider the subgroup

$$
U(2 n, \mathbb{R}):=S p(n, \mathbb{R}) \cap O(2 n, \mathbb{R}) \simeq U(n)
$$

2010 Mathematics Subject Classification. 42B35,22C05.

Key words and phrases. modulation spaces, metaplectic operators, symplectic group, unitary group, short-time Fourier transform. 
of symplectic rotations (cf., e.g. [15, Section 2.3]), namely

$$
U(2 n, \mathbb{R})=\left\{\left(\begin{array}{cc}
A & -B \\
B & A
\end{array}\right): A A^{T}+B B^{T}=I_{n}, A B^{T}=B^{T} A\right\} \subset S p(n, \mathbb{R})
$$

endowed with the normalized Haar measure $d S$ (the group $U(2 n, \mathbb{R})$, being compact, is unimodular).

In the 80's H. Feichtinger [16] introduced modulation spaces to measure the time-frequency concentration of a function/distribution on the time-frequency space (or phase space) $\mathbb{R}^{2 n}$. They are nowadays become popular among mathematicians and engineers because they have found numerous applications in signal processing [6, 17, 18], pseudodifferential and Fourier integral operators [7, 8, 9, 26, 27], partial differential equations [1, 2, 3, 4, 10, 13, 11, 11, 28, 29, 30] and quantum mechanics [12, 15].

To recall their definition, we need a few time-frequency tools. First, the translation $T_{x}$ and modulation $M_{\xi}$ operators are defined by

$$
T_{x} f(t)=f(t-x), \quad M_{\xi} f(t)=e^{2 \pi i t \cdot \xi} f(t), \quad t, x, \xi \in \mathbb{R}^{n},
$$

for any function $f$ on $\mathbb{R}^{n}$.

The time-frequency representation which occurs in the definition of modulation spaces is the short-time Fourier Transform (STFT) of a distribution $f \in \mathcal{S}^{\prime}\left(\mathbb{R}^{n}\right)$ with respect to a function $g \in \mathcal{S}\left(\mathbb{R}^{n}\right) \backslash\{0\}$ (so-called window), given by

$$
V_{g} f(x, \xi)=\left\langle f, M_{\xi} T_{x} g\right\rangle=\int_{\mathbb{R}^{n}} f(t) \overline{g(t-x)} e^{-2 \pi i t \cdot \xi} d t, \quad x, \xi \in \mathbb{R}^{n} .
$$

The short-time Fourier transform is well-defined whenever the bracket $\langle\cdot, \cdot\rangle$ makes sense for dual pairs of function or distribution spaces, in particular for $f \in$ $\mathcal{S}^{\prime}\left(\mathbb{R}^{n}\right), g \in \mathcal{S}\left(\mathbb{R}^{n}\right)$, or for $f, g \in L^{2}\left(\mathbb{R}^{n}\right)$.

Definition 1.1 (Modulation spaces). Given $g \in \mathcal{S}\left(\mathbb{R}^{n}\right)$, and $1 \leq p \leq \infty$, the modulation space $M^{p}\left(\mathbb{R}^{n}\right)$ consists of all tempered distributions $f \in \mathcal{S}^{\prime}\left(\mathbb{R}^{n}\right)$ such that $V_{g} f \in L^{p}\left(\mathbb{R}^{2 n}\right)$. The norm on $M^{p}\left(\mathbb{R}^{n}\right)$ is

$$
\|f\|_{M^{p}}=\left\|V_{g} f\right\|_{L^{p}}=\left(\int_{\mathbb{R}^{2 n}}\left|V_{g} f(x, \xi)\right|^{p} d x d \xi\right)^{1 / p}=\left(\int_{\mathbb{R}^{2 n}}\left|\left\langle f, M_{\xi} T_{x} g\right\rangle\right|^{p} d x d \xi\right)^{1 / p}
$$

(with obvious modifications for $p=\infty$ ).

The spaces $M^{p}\left(\mathbb{R}^{n}\right)$ are Banach spaces, and every nonzero $g \in M^{1}\left(\mathbb{R}^{n}\right)$ yields an equivalent norm in (44), so that their definition is independent of the choice of $g \in M^{1}\left(\mathbb{R}^{n}\right)$ (see [16, 20]).

We now provide an equivalent norm to (41) by using translations $T_{x}$ (or modulations $\left.M_{\xi}\right)$ and the operators $\widehat{S}$, with $S \in U(2 n, \mathbb{R})$ as follows. 
Theorem 1.2. Consider the Gaussian function $\varphi(t)=2^{d / 4} e^{-\pi|t|^{2}}$.

(i) For $1 \leq p<\infty$ and $f \in M^{p}\left(\mathbb{R}^{n}\right)$, we have

$$
\|f\|_{M^{p}\left(\mathbb{R}^{n}\right)} \asymp\left(\int_{\mathbb{R}^{n} \times U(2 n, \mathbb{R})}|x|^{n}\left|\left\langle f, \widehat{S} T_{x} \varphi\right\rangle\right|^{p} d x d S\right)^{\frac{1}{p}},
$$

where $d x$ is the Lebesgue measure on $\mathbb{R}^{n}$ and $d S$ the Haar measure on $U(2 n, \mathbb{R})$.

Similarly,

$$
\|f\|_{M^{p}\left(\mathbb{R}^{n}\right)} \asymp\left(\int_{\mathbb{R}^{n} \times U(2 n, \mathbb{R})}|\xi|^{n}\left|\left\langle f, \widehat{S} M_{\xi} \varphi\right\rangle\right|^{p} d \xi d S\right)^{\frac{1}{p}},
$$

with $d \xi$ being the Lebesgue measure on $\mathbb{R}^{n}$ and $d S$ the Haar measure on $U(2 n, \mathbb{R})$.

(ii) For $p=\infty, f \in M^{\infty}\left(\mathbb{R}^{n}\right)$, it occurs

$$
\|f\|_{M^{\infty}\left(\mathbb{R}^{n}\right)} \asymp \sup _{S \in U(2 n, \mathbb{R})} \sup _{x \in \mathbb{R}^{n}}\left|\left\langle f, \widehat{S} T_{x} \varphi\right\rangle\right|
$$

or, similarly,

$$
\|f\|_{M^{\infty}\left(\mathbb{R}^{n}\right)} \sup _{S \in U(2 n, \mathbb{R})} \sup _{\xi \in \mathbb{R}^{n}}\left|\left\langle f, \widehat{S} M_{\xi} \varphi\right\rangle\right| .
$$

The interpretation of the integral (5) above is as follows. The metaplectic operator $\widehat{S}$ produces a time-frequency rotation of the shifted Gaussian $T_{x} \varphi$. In this way, the operator

$$
f \mapsto\left\langle f, \widehat{S} T_{x} \varphi\right\rangle
$$

detects the time-frequency content of $f$ in an oblique strip, see Figure 1. All the contributions are then added together with a weight $|x|^{n}$ which takes into account the underlapping of the strips as $|x| \rightarrow \infty$ and the overlapping as $|x| \rightarrow 0$.

Formulas (6), (7) and (8) have similar meanings.

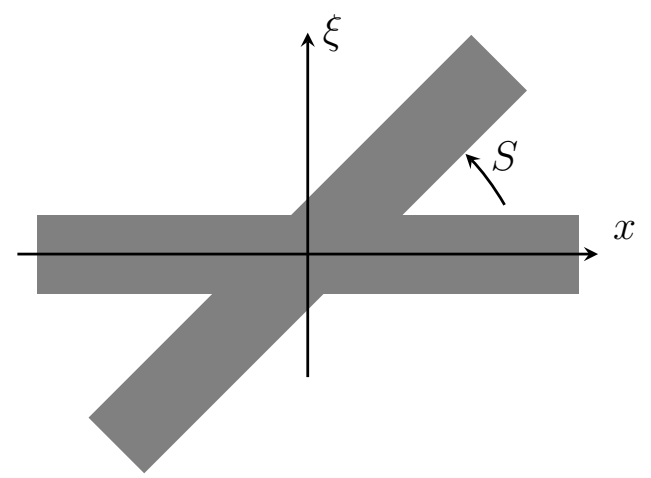

Figure 1. The time-frequency content of $f$ in the oblique strip is detected by the operator $f \mapsto\left\langle f, \widehat{S} T_{x} \varphi\right\rangle$ 
Observe that in dimension $n=1, U(2, \mathbb{R}) \simeq U(1)$ and the above formula is essentially a transition to polar coordinates with $|x|$ being the Jacobian.

Comparing (44) and (5) we observe that in (5) the modulation operator $M_{\xi}$ is replaced by the metaplectic operator $\widehat{S}$ and the integral on the phase space $\mathbb{R}^{2 n}$ has become an integral on the cartesian product $\mathbb{R}^{n} \times U(2 n, \mathbb{R})$. The integration parameters $(x, \xi)$ of (4) live in $\mathbb{R}^{2 n}$, with $\operatorname{dim} \mathbb{R}^{2 n}=2 n$, whereas the parameters $(x, S)$ of (15) live in $\mathbb{R}^{n} \times U(2 n, \mathbb{R})$. Recall that $\operatorname{dim} U(2 n, \mathbb{R})=n^{2}[15]$; this suggests that a formula similar to (5) should hold when $U(2 n, \mathbb{R})$ is reduced to a suitable subgroup $K \subset U(2 n, \mathbb{R})$ of dimension $n$. This is indeed the case, as shown in the subsequent Theorem 1.3 .

Consider the $n$-dimensional torus

$$
\mathbb{T}^{n}=\left\{S=\left(\begin{array}{ccc}
e^{i \theta_{1}} & & \\
& \ddots & \\
& & e^{i \theta_{n}}
\end{array}\right): \theta_{1}, \ldots, \theta_{n} \in \mathbb{R}\right\} \subset U(n)
$$

with the Haar measure $d S=d \theta_{1} \ldots d \theta_{n}$. The torus is isomorphic to a subgroup $K \subset U(2 n, \mathbb{R})$, via the isomorphism $\iota$ in formula (16) below (see the subsequent section).

We exhibit the following characterization for $M^{p}$-spaces.

Theorem 1.3. Let $\varphi$ be the Gaussian of Theorem 1.2.

(i) For $1 \leq p<\infty, f \in M^{p}\left(\mathbb{R}^{n}\right)$, we have

$$
\|f\|_{M^{p}\left(\mathbb{R}^{n}\right)} \asymp\left(\int_{\mathbb{R}^{n} \times \mathbb{T}^{n}}\left|x_{1} \ldots x_{n} \|\left\langle f, \widehat{S} T_{x} \varphi\right\rangle\right|^{p} d x d S\right)^{\frac{1}{p}},
$$

or, similarly,

$$
\|f\|_{M^{p}\left(\mathbb{R}^{n}\right)} \asymp\left(\int_{\mathbb{R}^{n} \times \mathbb{T}^{n}}\left|\xi_{1} \ldots \xi_{n} \|\left\langle f, \widehat{S} M_{\xi} \varphi\right\rangle\right|^{p} d \xi d S\right)^{\frac{1}{p}} .
$$

(ii) For $p=\infty$,

$$
\|f\|_{M^{\infty}\left(\mathbb{R}^{n}\right)} \asymp \sup _{S \in \mathbb{T}^{n}} \sup _{x \in \mathbb{R}^{n}}\left|\left\langle f, \widehat{S} T_{x} \varphi\right\rangle\right|
$$

or

$$
\|f\|_{M^{\infty}\left(\mathbb{R}^{n}\right)} \asymp \sup _{S \in \mathbb{T}^{n}} \sup _{\xi \in \mathbb{R}^{n}}\left|\left\langle f, \widehat{S} M_{\xi} \varphi\right\rangle\right| .
$$

The above results for the groups $U(2 n, \mathbb{R})$ and $\mathbb{T}^{n}$ can be interpreted, in a sense, as two extreme cases, and it would be interesting to find, more generally, for which compact subgroups $K \subset U(2 n, \mathbb{R})$ similar characterizations hold. We conjecture that they should be precisely the subgroups $K \subset U(2 n, \mathbb{R})$ such that every orbit for their action on $\mathbb{R}^{2 n}$ intersects $\{0\} \times \mathbb{R}^{n}$ (up to subsets of measure zero), with a corresponding weighted measure on $\mathbb{R}^{n} \times K$ to be determined. 
Another open problem which is worth investigating is the study of discrete versions of the above characterizations.

The paper is organized as follows: in Section 2 we collected some preliminary results, whereas Section 3 is devoted to the proof of Theorems 1.2 and 1.3 . In Section 4 we rephrase more explicitly Theorem 1.3 in terms of the partial fractional Fourier transform.

\section{Notation And Preliminaries}

Notation. We write $x \cdot y$ for the scalar product on $\mathbb{R}^{n}$ and $|t|^{2}=t \cdot t$, for $t, x, y \in \mathbb{R}^{n}$. For expressions $A, B \geq 0$, we use the notation $A \lesssim B$ to represent the inequality $A \leq c B$ for a suitable constant $c>0$, and $A \asymp B$ for the equivalence $c^{-1} B \leq A \leq c B$.

The Schwartz class is denoted by $\mathcal{S}\left(\mathbb{R}^{n}\right)$, the space of tempered distributions by $\mathcal{S}^{\prime}\left(\mathbb{R}^{n}\right)$. We use the brackets $\langle f, g\rangle$ to denote the extension to $\mathcal{S}^{\prime}\left(\mathbb{R}^{n}\right) \times \mathcal{S}\left(\mathbb{R}^{n}\right)$ of the inner product $\langle f, g\rangle=\int f(t) \overline{g(t)} d t$ on $L^{2}\left(\mathbb{R}^{n}\right)$.

Metaplectic Operators. The metaplectic representation $\mu$ of $M p(n, \mathbb{R})$, the two-sheeted cover of the symplectic group $S p(n, \mathbb{R})$, defined in (1) arises as intertwining operator between the standard Schrödinger representation $\rho$ of the Heisenberg group $\mathbb{H}^{d}$ and the representation that is obtained from it by composing $\rho$ with the action of $S p(n, \mathbb{R})$ by automorphisms on $\mathbb{H}^{d}$ (see, e.g., $[15,[19,21]$ ). Let us recall the main points of a direct construction.

The symplectic group $S p(n, \mathbb{R})$ is generated by the so-called free symplectic matrices

$$
S=\left(\begin{array}{ll}
A & B \\
C & D
\end{array}\right) \in S p(n, \mathbb{R}), \quad \operatorname{det} B \neq 0 .
$$

To each such a matrix the associated generating function is defined by

$$
W\left(x, x^{\prime}\right)=\frac{1}{2} D B^{-1} x \cdot x-B^{-1} x \cdot x^{\prime}+\frac{1}{2} B^{-1} A x^{\prime} \cdot x^{\prime} .
$$

Conversely, to every polynomial of the type

$$
W\left(x, x^{\prime}\right)=\frac{1}{2} P x \cdot x-L x \cdot x^{\prime}+\frac{1}{2} Q x^{\prime} \cdot x^{\prime}
$$

with

$$
P=P^{T}, Q=Q^{T}
$$

and

$$
\operatorname{det} L \neq 0
$$

it can be associated a free symplectic matrix, namely

$$
S_{W}=\left(\begin{array}{cc}
L^{-1} Q & L^{-1} \\
P L^{-1} Q-L^{T} & P L^{-1}
\end{array}\right) .
$$


Given $S_{W}$ as above and $m \in \mathbb{Z}$ such that

$$
m \pi \equiv \arg \operatorname{det} L \bmod 2 \pi,
$$

the related operator $\widehat{S}_{W, m}$ is defined by setting, for $\psi \in \mathcal{S}\left(\mathbb{R}^{n}\right)$,

$$
\widehat{S}_{W, m} \psi(x)=\frac{1}{i^{n / 2}} \Delta(W) \int_{\mathbb{R}^{n}} e^{2 \pi i W\left(x, x^{\prime}\right)} \psi\left(x^{\prime}\right) d x^{\prime}
$$

$\left(\right.$ with $\left.i^{n / 2}=e^{i \pi n / 4}\right)$ where

$$
\Delta(W)=i^{m} \sqrt{|\operatorname{det} L|} .
$$

The operator $\widehat{S}_{W, m}$ is named quadratic Fourier transform associated to the free symplectic matrix $S_{W}$. The class modulo 4 of the integer $m$ is called Maslov index of $\widehat{S}_{W, m}$. Observe that if $m$ is one choice of Maslov index, then $m+2$ is another equally good choice: hence to each function $W$ we associate two operators, namely $\widehat{S}_{W, m}$ and $\widehat{S}_{W, m+2}=-\widehat{S}_{W, m}$.

The quadratic Fourier transform corresponding to the choices $S_{W}=J$ and $m=0$ is denoted by $\widehat{J}$. The generating function of $J$ is simply $W\left(x, x^{\prime}\right)=-x \cdot x^{\prime}$. It follows that

$$
\widehat{J} \psi(x)=\frac{1}{i^{n / 2}} \int_{\mathbb{R}^{n}} e^{-2 \pi i x \cdot x^{\prime}} \psi\left(x^{\prime}\right) d x^{\prime}=\frac{1}{i^{n / 2}} \mathcal{F} \psi(x)
$$

for $\psi \in \mathcal{S}\left(\mathbb{R}^{n}\right)$, where $\mathcal{F}$ is the usual unitary Fourier transform.

The quadratic Fourier transforms $\widehat{S}_{W, m}$ form a subset of the group $\mathcal{U}\left(L^{2}\left(\mathbb{R}^{n}\right)\right)$ of unitary operators acting on $L^{2}\left(\mathbb{R}^{n}\right)$, which is closed under the operation of inversion and they generate a subgroup of $\mathcal{U}\left(L^{2}\left(\mathbb{R}^{n}\right)\right)$ which is, by definition, the metaplectic group $M p(n, \mathbb{R})$. The elements of $M p(n, \mathbb{R})$ are called metaplectic operators.

Hence, every $\widehat{S} \in M p(n, \mathbb{R})$ is, by definition, a product

$$
\widehat{S}_{W_{1}, m_{1}} \ldots \widehat{S}_{W_{k}, m_{k}}
$$

of metaplectic operators associated to free symplectic matrices.

Indeed, it can be proved that every $\widehat{S} \in M p(n, \mathbb{R})$ can be written as a product of exactly two quadratic Fourier transforms: $\widehat{S}=\widehat{S}_{W, m} \widehat{S}_{W^{\prime}, m^{\prime}}$. Now, it can be shown that the mapping

$$
\widehat{S}_{W, m} \longmapsto S_{W}
$$

extends to a group homomorphism

$$
\pi: M p(n, \mathbb{R}) \rightarrow S p(n, \mathbb{R}),
$$

which is in fact a double covering.

We also observe that each metaplectic operator is, by construction, a unitary operator in $L^{2}\left(\mathbb{R}^{n}\right)$, but also an automorphism of $\mathcal{S}\left(\mathbb{R}^{n}\right)$ and of $\mathcal{S}^{\prime}\left(\mathbb{R}^{n}\right)$. 
We are interested in its restriction $\widehat{S}=\pi(S)$, with $S \in U(2 n, \mathbb{R})$, the symplectic rotations in (2).

Observe that $U(n):=U(n, \mathbb{C})$, the complex unitary group (the group of $n \times n$ invertible complex matrices $V$ satisfying $\left.V V^{*}=V^{*} V=I_{n}\right)$ is isomorphic to $U(2 n, \mathbb{R})$. The isomorphism $\iota$ is the mapping $\iota: U(n) \rightarrow U(2 n, \mathbb{R})$ given by

$$
\iota(A+i B)=\left(\begin{array}{cc}
A & -B \\
B & A
\end{array}\right),
$$

for details see [15, Chapter 2.3].

We present here some results related to the group $U(2 n, \mathbb{R})$, which will be used in the sequel to attain the characterization of Theorem 1.2. First, we recall a well-known result, see for instance [20, Lemma 9.4.3]:

Lemma 2.1. For $f, g \in L^{2}\left(\mathbb{R}^{n}\right)$ and $S \in S p(n, \mathbb{R})$, the STFT $V_{g} f$ satisfies

$$
\left|V_{\widehat{S} g}(\widehat{S} f)(x, \xi)\right|=\left|V_{g} f\left(S^{-1}(x, \xi)\right)\right|, \quad(x, \xi) \in \mathbb{R}^{2 n} .
$$

This second issue is contained in [5], we sketch the proof for the sake of consistency.

Lemma 2.2. For $\varphi, \psi \in \mathcal{S}\left(\mathbb{R}^{n}\right)$ and $S \in U(2 n, \mathbb{R})$, the $\operatorname{STFT} V_{\varphi}(\widehat{S} \psi)$ is a Schwartz function, with seminorms uniformly bounded when $S \in U(2 n, \mathbb{R})$.

Proof. Since $\varphi \in \mathcal{S}\left(\mathbb{R}^{n}\right)$, the $\mathrm{STFT} V_{\varphi}$ is a continuous mapping from $\mathcal{S}\left(\mathbb{R}^{n}\right)$ into $\mathcal{S}\left(\mathbb{R}^{2 n}\right)$ (see [16]). Hence, it is enough to show that

$$
\{\hat{S} \varphi: S \in U(2 n, \mathbb{R})\}
$$

is a bounded subset of the Schwartz class $\mathcal{S}\left(\mathbb{R}^{n}\right)$, i.e., every Schwartz seminorm is bounded on it. Since the group $U(2 n, \mathbb{R})$ is compact, it is sufficient to show that every seminorm is locally bounded, that is, we can limit ourselves to consider $S$ in a sufficiently small neighbourhood for any fixed $S_{0} \in U(2 n, \mathbb{R})$. Equivalently, we can consider $S$ of the form $S=S_{1} J^{-1} S_{0}$ where $S_{1}$ belongs to a enough small neighbourhood of $J$ in $U(2 n, \mathbb{R})$. Using the representation of metaplectic operators recalled at the beginning of this section, we can write

$$
\begin{aligned}
\hat{S} \varphi(x) & = \pm \widehat{S}_{1}\left[\widehat{J}^{-1} \widehat{S}_{0} \varphi\right](x) \\
& =c \sqrt{|\operatorname{det} L|} \int_{\mathbb{R}^{n}} e^{2 \pi i\left(\frac{1}{2} P x \cdot x-L x \cdot y+\frac{1}{2} Q y \cdot y\right)}[\underbrace{\left.\widehat{J}^{-1} \widehat{S}_{0} \varphi\right]}_{\in \mathcal{S}\left(\mathbb{R}^{n}\right)}(y) d y
\end{aligned}
$$

where $|c|=1$ and, we might say, $\|P\|<\epsilon,\|Q\|<\epsilon,\|L-I\|<\epsilon$. If $\epsilon<1$, it is straightforward to check that $\hat{S} \varphi$ belongs to a bounded subset of $\mathcal{S}\left(\mathbb{R}^{n}\right)$, as desired. 
Lemma 2.3. Let $B=\left(b_{i, j}\right)_{i, j=1, \ldots n}$ be the $n \times n$ submatrix in (2). The subset $\Sigma \subset U(2 n, \mathbb{R})$ obtained by setting $b_{i, 1}=0, i=1, \ldots n$ (i.e., the first column of $B$ is set to zero), is a submanifold of codimension $n$.

Proof. We have to verify that the coordinates $b_{1,1}, \ldots, b_{n, 1}$ are independent on the subset $\Sigma$, namely the projection

$$
\left(b_{1,1}, \ldots, b_{n, 1}\right): U(2 n, \mathbb{R}) \rightarrow \mathbb{R}^{n}
$$

has rank $n$ on $\Sigma$.

Let us first show that for every $S_{0} \in \Sigma$ there exists a $U(2 n, \mathbb{R})$-valued smooth function $S\left(b_{1}, \ldots, b_{n}\right)$, defined in a neighbourhood of $0 \in \mathbb{R}^{n}$, such that $S(0)=S_{0}$ and the first column "of its submatrix $B$ " is precisely $\left(b_{1}, \ldots, b_{n}\right)^{T}$.

Let $S_{0}=A+i B=\left(V_{1}, \ldots, V_{n}\right) \in \Sigma$, with $V_{j}$ being a $n \times 1$ complex vector, $j=1, \ldots, n$, so that by assumption $\left(b_{i, 1}\right)_{i=1, \ldots, n}=\operatorname{Im} V_{1}=0$. We consider any smooth function $V_{1}\left(b_{1}, \ldots, b_{n}\right)$, defined in a neighbourhood of $0 \in \mathbb{R}^{n}$, valued in the unit sphere of $\mathbb{C}^{n}$, such that

$$
\operatorname{Im} V_{1}\left(b_{1}, \ldots, b_{n}\right)=\left(b_{1}, \ldots, b_{n}\right)^{T}, \quad V_{1}(0)=V_{1} .
$$

Then, we apply the Gram-Schmidt orthonormalization procedure in $\mathbb{C}^{n}$ to the set of vectors $\left(V_{1}\left(b_{1}, \ldots, b_{n}\right), V_{2}, \ldots, V_{n}\right)$. This provides the desired $U(n)$-valued function $S\left(b_{1}, \ldots, b_{n}\right)$. In particular $S(0)=S_{0}$.

Now, the composition of the mapping

$$
\left(b_{1}, \ldots, b_{n}\right) \mapsto S\left(b_{1}, \ldots, b_{n}\right)
$$

followed by the projection $\left(b_{1,1}, \ldots, b_{n, 1}\right): U(2 n, \mathbb{R}) \rightarrow \mathbb{R}^{n}$ is therefore the identity mapping in a neighbourhood of 0 and has rank $n$. Hence the same is true for the projection $\left(b_{1,1}, \ldots, b_{n, 1}\right): U(2 n, \mathbb{R}) \rightarrow \mathbb{R}^{n}$ at $S_{0}$.

Lemma 2.4. For every $\epsilon>0$, define

$$
\chi_{\epsilon}(x, \xi)=\frac{1}{\epsilon^{n}} \mathbb{1}_{Q}\left(\frac{\xi}{\epsilon}\right),
$$

where

$$
Q=\left[-\frac{1}{2}, \frac{1}{2}\right]^{n} \subset \mathbb{R}^{n} \text { and } \mathbb{1}_{Q}= \begin{cases}1, & \xi \in Q \\ 0, & \xi \notin Q\end{cases}
$$

and

$$
\tilde{\chi}_{\epsilon}(z)=\frac{\chi_{\epsilon}(z)}{\int_{U(2 n, \mathbb{R})} \chi_{\epsilon}(S z) d S}, \quad z \in \mathbb{R}^{2 n} .
$$

Then we have

$$
\int_{U(2 n, \mathbb{R})} \tilde{\chi}_{\epsilon}(S z) d S=1, \quad \forall z \in \mathbb{R}^{2 n}
$$


A CHARACTERIZATION OF MODULATION SPACES BY SYMPLECTIC ROTATIONS 9

and

$$
\lim _{\epsilon \rightarrow 0^{+}} \int_{\mathbb{R}^{2 n}} \tilde{\chi}_{\epsilon}(x, \xi) \Phi(x, \xi) d x d \xi=C \int_{\mathbb{R}^{n}}|x|^{n} \Phi(x, 0) d x
$$

for some $C>0$ and for every continuous function $\Phi$ on $\mathbb{R}^{2 n}$ with a rapid decay at infinity.

Proof. We will show in a moment that, for $z=(x, \xi) \in \mathbb{R}^{2 n}$,

$$
\int_{U(2 n, \mathbb{R})} \chi_{\epsilon}(S z) d S \gtrsim \min \left\{\epsilon^{-n},|z|^{-n}\right\}
$$

(with the convention, at $z=0$, that $\min \left\{\epsilon^{-n},+\infty\right\}=\epsilon^{-n}$ ). In particular, $\int_{U(2 n, \mathbb{R})} \chi_{\epsilon}(S z) d S \neq 0$, for every $z \in \mathbb{R}^{2 n}$. Formula (20) then follows, because

$$
\begin{aligned}
\int_{U(2 n, \mathbb{R})} \tilde{\chi}_{\epsilon}(S z) d S & =\int_{U(2 n, \mathbb{R})} \frac{\chi_{\epsilon}(S z)}{\int_{U(2 n, \mathbb{R})} \chi_{\epsilon}(U S z) d U} d S \\
& =\int_{U(2 n, \mathbb{R})} \frac{\chi_{\epsilon}(S z)}{\int_{U(2 n, \mathbb{R})} \chi_{\epsilon}(U z) d U} d S=1
\end{aligned}
$$

for every $z \in \mathbb{R}^{2 n}$, since the Haar measure is right invariant.

Let us now prove (22). For $z=0$ we have

$$
\int_{U(2 n, \mathbb{R})} \chi_{\epsilon}(S z) d S=\frac{1}{\epsilon^{n}} \int_{U(2 n, \mathbb{R})} d S=\frac{C_{0}}{\epsilon^{n}},
$$

with $C_{0}=\operatorname{meas}(U(2 n, \mathbb{R}))>0$. Consider now $z \neq 0$. Observe that the function

$$
\Psi_{\epsilon}(z):=\int_{U(2 n, \mathbb{R})} \chi_{\epsilon}(S z) d S
$$

is constant on the orbits of $U(2 n, \mathbb{R})$ in $\mathbb{R}^{2 n}$, so that we can suppose

$$
z=(x, 0), \quad x=\left(x_{1}, 0, \ldots, 0\right), \quad x_{1}=|x|=|z|>0 .
$$

Now, by the definition of $\chi_{\epsilon}$ and $\Psi_{\epsilon}$,

$$
\Psi_{\epsilon}(z)=\epsilon^{-n} \text { meas }\left\{S=\left(\begin{array}{cc}
A & -B \\
B & A
\end{array}\right) \in U(2 n, \mathbb{R}):\left|b_{i, 1}\right|<\frac{\epsilon}{2|z|}, i=1, \ldots, n\right\},
$$

where $\left(b_{i, 1}\right)_{i=1, \ldots, n}$, is the first column of the matrix $B=\left(b_{i, j}\right)_{i, j=1, \ldots n}$.

Define, for $\mu>0$,

$$
f(\mu)=\operatorname{meas}\left\{S=\left(\begin{array}{cc}
A & -B \\
B & A
\end{array}\right) \in U(2 n, \mathbb{R}):\left|b_{i, 1}\right|<\mu, i=1, \ldots, n\right\} .
$$

Observe that $f(\mu)$ is non-decreasing and constant for $\mu \geq 1$. Moreover, from Lemma 2.3 we know that by setting $b_{i, 1}=0, i=1, \ldots, n$, in $U(2 n, \mathbb{R})$, we get a submanifold $\Sigma$ of codimension $n$, and the function $f(\mu)$ is the measure 
of a tubular neighbourhood of $\Sigma$ in $U(2 n, \mathbb{R})$. Hence we have the asymptotic behaviour

$$
\mu^{-n} f(\mu) \rightarrow C_{0}>0, \quad \text { as } \quad \mu \rightarrow 0^{+}
$$

and in particular

$$
f(\mu) \gtrsim \min \left\{1, \mu^{n}\right\}
$$

We then infer

$$
\Psi_{\epsilon}(z)=\epsilon^{-n} f\left(\frac{\epsilon}{2|z|}\right) \rightarrow \frac{C_{1}}{|z|^{n}}, \quad \text { as } \epsilon \rightarrow 0^{+}
$$

locally uniformly in $\mathbb{R}^{2 n} \backslash\{0\}$, with $C_{1}=2^{-n} C_{0}$, and

$$
\Psi_{\epsilon}(z) \gtrsim \epsilon^{-n} \min \left\{1,\left(\frac{\epsilon}{|z|}\right)^{n}\right\}=\min \left\{\epsilon^{-n},|z|^{-n}\right\}
$$

which is (22).

Let us finally prove (21). We are interested in the limit $\epsilon \rightarrow 0^{+}$, so we can assume $\epsilon \leq 1$. Consider a continuous function $\Phi$ on $\mathbb{R}^{2 n}$ with rapid decay at infinity. By definition of $\tilde{\chi}_{\epsilon}(z)$ in (19) we have

$$
\tilde{\chi}_{\epsilon}(x, \xi)=\frac{\epsilon^{-n}}{\Psi_{\epsilon}(x, \xi)} \mathbb{1}_{[-\epsilon / 2, \epsilon / 2]^{n}}(\xi)
$$

so that, by (27),

$$
\left|\tilde{\chi}_{\epsilon}(x, \xi) \Phi(x, \xi)\right| \lesssim \epsilon^{-n}\left(1+|x|^{n}\right) \mathbb{1}_{[-\epsilon / 2, \epsilon / 2]^{n}}(\xi)|\Phi(x, \xi)| \in L^{1}\left(\mathbb{R}^{2 n}\right)
$$

for $0<\epsilon \leq 1$. Fubini's Theorem then allows one to look at the first integral in (21) as an iterated integral

$$
I_{\epsilon}:=\int_{\mathbb{R}^{n}}\left(\int_{\mathbb{R}^{n}} \tilde{\chi}_{\epsilon}(x, \xi) \Phi(x, \xi) d \xi\right) d x
$$

and we apply the dominated convergence theorem to the integral with respect to the $x$ variable as follows. Setting

$$
\Upsilon_{\epsilon}(x):=\int_{\mathbb{R}^{n}} \tilde{\chi}_{\epsilon}(x, \xi) \Phi(x, \xi) d \xi=\epsilon^{-n} \int_{[-\epsilon / 2, \epsilon / 2]^{n}} \frac{1}{\Psi_{\epsilon}(x, \xi)} \Phi(x, \xi) d \xi,
$$

by (26) we have, for every fixed $x \neq 0$,

$$
\Upsilon_{\epsilon}(x) \rightarrow C|x|^{n} \Phi(x, 0) ;
$$

for some constant $C>0$. On the other hand $\Upsilon_{\epsilon}(x)$ is dominated, using (27), by

$$
(1+|x|)^{n} \sup _{\xi \in \mathbb{R}^{n}}|\Phi(x, \xi)| \in L^{1}\left(\mathbb{R}^{n}\right) .
$$

Hence

$$
\lim _{\epsilon \rightarrow 0^{+}} I_{\epsilon}=\int_{\mathbb{R}^{n}} \lim _{\epsilon \rightarrow 0^{+}} \Upsilon_{\epsilon}(x) d x=C \int_{\mathbb{R}^{n}}|x|^{n} \Phi(x, 0) d x
$$


This concludes the proof.

Remark 2.5. Observe that there are no conditions on the derivatives of the function $\Phi$ in (21).

\section{Proofs of the main Results}

In what follows we prove Theorems 1.2 and 1.3 .

Proof of Theorem 1.2. (i) First Step. Let us start with showing that formula (5) is true for any function $\psi$ in the Schwartz class $\mathcal{S}\left(\mathbb{R}^{n}\right) \subset M^{p}\left(\mathbb{R}^{n}\right), 1 \leq p<\infty$. Using the Gaussian $\varphi(t)=2^{d / 4} e^{-\pi|t|^{2}}$ as window function, we compute the $M^{p_{-}}$ norm of $\psi$ as in (4) and then use Lemma 2.4 so that

$$
\begin{aligned}
\|\psi\|_{M^{p}}^{p} & =\int_{\mathbb{R}^{2 n}}\left|V_{\varphi} \psi(z)\right|^{p} d z=\int_{\mathbb{R}^{2 n}} \int_{U(2 n, \mathbb{R})} \tilde{\chi}_{\epsilon}(S z)\left|V_{\varphi} \psi(z)\right|^{p} d S d z \\
& =\int_{\mathbb{R}^{2 n}} \int_{U(2 n, \mathbb{R})} \tilde{\chi}_{\epsilon}(z)\left|V_{\varphi} \psi\left(S^{-1} z\right)\right|^{p} d S d z \\
& =\int_{\mathbb{R}^{2 n}} \int_{U(2 n, \mathbb{R})} \tilde{\chi}_{\epsilon}(z)\left|V_{\widehat{S} \varphi} \widehat{S} \psi(z)\right|^{p} d S d z
\end{aligned}
$$

where in the last equality we used Lemma 2.1. Observe that, since $S$ is unitary and $\varphi$ is a Gaussian, $\widehat{S} \varphi=c \varphi$, for some phase factor $c \in \mathbb{C}$, with $|c|=1$ (see [15, Proposition 252]) and this phase factor is killed by the modulus obtaining $\left|V_{\widehat{S} \varphi} \widehat{S} \psi(z)\right|=\left|V_{\varphi} \widehat{S} \psi(z)\right|$. Continuing the above computation we infer

$$
\|\psi\|_{M^{p}}^{p}=\int_{\mathbb{R}^{2 n}} \tilde{\chi}_{\epsilon}(z) \int_{U(2 n, \mathbb{R})}\left|V_{\varphi} \widehat{S} \psi(z)\right|^{p} d S d z
$$

Set

$$
\Phi(z)=\int_{U(2 n, \mathbb{R})}\left|V_{\varphi} \widehat{S} \psi(z)\right|^{p} d S .
$$

The dominated convergence theorem guarantees that $\Phi$ is continuous on $\mathbb{R}^{2 n}$, moreover $\Phi$ has rapid decay at infinity. This follows from Lemma 2.2.

Letting $\epsilon \rightarrow 0^{+}$and using (21) we obtain

$$
\begin{aligned}
\|\psi\|_{M^{p}}^{p} & =C \int_{\mathbb{R}^{n}}|x|^{n} \int_{U(2 n, \mathbb{R})}\left|V_{\varphi} \widehat{S} \psi(x, 0)\right|^{p} d S d x \\
& =C \int_{\mathbb{R}^{n}}|x|^{n} \int_{U(2 n, \mathbb{R})}\left|\left\langle\widehat{S} \psi, T_{x} \varphi\right\rangle\right|^{p} d S d x \\
& =C \int_{\mathbb{R}^{n}}|x|^{n} \int_{U(2 n, \mathbb{R})}\left|\left\langle\psi, \widehat{S} T_{x} \varphi\right\rangle\right|^{p} d S d x .
\end{aligned}
$$


The last equality is due to $\left\langle\widehat{S} \psi, T_{x} \varphi\right\rangle=\left\langle\psi, \widehat{S}^{-1} T_{x} \varphi\right\rangle$ and the invariance of the Haar measure with respect to the change of variable $S \rightarrow S^{-1}$.

Second Step. Consider $f \in M^{p}\left(\mathbb{R}^{n}\right), 1 \leq p<\infty$. Using the density of the Schwartz class $\mathcal{S}\left(\mathbb{R}^{n}\right)$ in $M^{p}\left(\mathbb{R}^{n}\right)$ (cf. e.g., [20, Chapter 12]), there exists a sequence $\left\{\psi_{k}\right\}_{k} \in \mathcal{S}\left(\mathbb{R}^{n}\right)$ such that $\psi_{k} \rightarrow f$ in $M^{p}\left(\mathbb{R}^{n}\right)$. This implies that $\psi_{k} \rightarrow f$ in $\mathcal{S}^{\prime}\left(\mathbb{R}^{n}\right)$ and

$$
\left\langle\psi_{k}, \widehat{S} T_{x} \varphi\right\rangle \rightarrow\left\langle\psi, \widehat{S} T_{x} \varphi\right\rangle
$$

pointwise for every $x \in \mathbb{R}^{n}, S \in U(2 n, \mathbb{R})$. Let us define, for every $f \in M^{p}\left(\mathbb{R}^{n}\right)$,

$$
\||f| \mid=\left(\int_{\mathbb{R}^{n} \times U(2 n, \mathbb{R})}|x|^{n}\left|\left\langle f, \widehat{S} T_{x} \varphi\right\rangle\right|^{p} d x d S\right)^{\frac{1}{p}} .
$$

By Fatou's Lemma, for any $f \in M^{p}\left(\mathbb{R}^{n}\right)$ :

$$
\||| f \mid\|^{p} \leq \liminf _{k \rightarrow \infty}\|\| \psi_{k}\left\|^{p} \lesssim \liminf _{k \rightarrow \infty}\right\| \psi_{k}\left\|_{M^{p}}^{p}=\right\| f \|_{M^{p}}^{p}
$$

It is easy to check that $\||f|\|$ is a seminorm on $M^{p}\left(\mathbb{R}^{n}\right)$. Applying (29) to the difference $f-\psi_{k}$ we obtain $\left\|\left|f-\psi_{k}\right|\right\| \rightarrow 0$ and hence $\left\|\left|\psi_{k}\|\| \rightarrow\||| f\|\right|\right.$. By assumption we also have $\left\|\psi_{k}\right\|_{M^{p}} \rightarrow\|f\|_{M^{p}}$, and the desired norm equivalence in (5) then extents from $\mathcal{S}\left(\mathbb{R}^{n}\right)$ to $M^{p}\left(\mathbb{R}^{n}\right)$.

Third Step. We will show that (66) easily follows from (5) . Indeed, the Fourier transform $\widehat{J}=\mathcal{F}$ is a metaplectic operator and we recall that the Fourier transform is a topological isomorphism $\mathcal{F}: M^{p}\left(\mathbb{R}^{n}\right) \rightarrow M^{p}\left(\mathbb{R}^{n}\right), 1 \leq p \leq \infty$, [16]. Furthermore, by the definition of the symplectic group (1), for any $S \in U(2 n, \mathbb{R})$,

$$
J^{-1} S=\left(S^{T}\right)^{-1} J^{-1}=S J^{-1}
$$

for $S^{-1}=S^{T}$. For any $f \in M^{p}\left(\mathbb{R}^{n}\right),\|f\|_{M^{p}} \asymp\|\hat{f}\|_{M^{p}}$, and using (15),

$$
\begin{aligned}
\left|\left\langle\hat{f}, S T_{x} \varphi\right\rangle\right| & =\left|\left\langle f, \widehat{J^{-1}} \widehat{S} T_{x} \varphi\right\rangle\right|=\left|\left\langle f, \widehat{S} \mathcal{F}^{-1} T_{x} \varphi\right\rangle\right| \\
& =\left|\left\langle f, \widehat{S} M_{x} \mathcal{F}^{-1} \varphi\right\rangle\right|=\left|\left\langle f, \widehat{S} M_{x} \varphi\right\rangle\right|
\end{aligned}
$$

since the Gaussian is an eigenvector of $\mathcal{F}^{-1}$ with eigenvalue equal to 1 . This immediately yields (6).

(ii) Case $p=\infty$. Observe that any $z \in \mathbb{R}^{2 n}$ can be written as

$$
z=S^{-1}\left(\begin{array}{l}
x \\
0
\end{array}\right)
$$


for some $x \in \mathbb{R}^{n}, S \in U(2 n, \mathbb{R})$, so that, for any $f \in M^{\infty}\left(\mathbb{R}^{n}\right)$,

$$
\begin{aligned}
\|f\|_{M^{\infty}\left(\mathbb{R}^{n}\right)} & =\sup _{z \in \mathbb{R}^{2 n}}\left|V_{\varphi} f(z)\right| \asymp \sup _{S \in U(2 n, \mathbb{R})} \sup _{x \in \mathbb{R}^{n}}\left|V_{\varphi} f\left(S^{-1}\left(\begin{array}{c}
x \\
0
\end{array}\right)\right)\right| \\
& =\sup _{S \in U(2 n, \mathbb{R})} \sup _{x \in \mathbb{R}^{n}}\left|V_{\varphi}(S f)(x, 0)\right|=\sup _{S \in U(2 n, \mathbb{R})} \sup _{x \in \mathbb{R}^{n}}\left|\left\langle S f, T_{x} \varphi\right\rangle\right| \\
& =\sup _{S \in U(2 n, \mathbb{R})} \sup _{x \in \mathbb{R}^{n}}\left|\left\langle f, S T_{x} \varphi\right\rangle\right|,
\end{aligned}
$$

as desired.

We now prove the similar result, with the group $U(2 n, \mathbb{R})$ replaced by the subgroup $\mathbb{T}^{n}$ (up to isomorphisms).

Proof of Theorem 1.3 . (i) The proof uses a similar pattern to Theorem 1.2, replacing the group $U(2 n, \mathbb{R})$ by $\mathbb{T}^{n}$. The preparation of Lemma 2.3 is no longer necessary. Lemma 2.4 must be revisited in this context as follows. Using the same notation, to estimate the function $\Psi_{\epsilon}(z)$ we again observe that it is constant on the orbits of the torus $\mathbb{T}^{n}$, so that we can suppose

$$
z=(x, 0), \quad x=\left(x_{1}, \ldots, x_{n}\right), \quad x_{j}=\left|x_{j}\right|=\left|z_{j}\right|>0, \quad j=1, \ldots, n .
$$

Then

$$
\begin{aligned}
\Psi_{\epsilon}(z) & =\epsilon^{-n} \text { meas }\left\{S=\left(\begin{array}{ccc}
e^{i \theta_{1}} & & \\
& \ddots & \\
& & e^{i \theta_{n}}
\end{array}\right):\left|\sin \theta_{j}\right|<\frac{\epsilon}{2\left|z_{j}\right|}, j=1, \ldots, n\right\} \\
& =\epsilon^{-n} f\left(\frac{\epsilon}{2\left|z_{1}\right|}\right) \ldots f\left(\frac{\epsilon}{2\left|z_{n}\right|}\right),
\end{aligned}
$$

where now we set, for $\mu>0$,

$$
f(\mu)=\operatorname{meas}\{\theta \in[0,2 \pi]:|\sin \theta|<\mu\} .
$$

We have

$$
\mu^{-1} f(\mu) \rightarrow C>0 \quad \text { as } \quad \epsilon \rightarrow 0^{+}
$$

and

$$
f(\mu) \gtrsim \min \{1, \mu\}
$$

which gives

$$
\Psi_{\epsilon}(z) \rightarrow \frac{C}{\left|z_{1} \cdots z_{n}\right|}, \quad \text { as } \quad \epsilon \rightarrow 0^{+}
$$

locally uniformly for $z_{1}, \ldots, z_{n} \in \mathbb{R}^{2} \backslash\{0\}$, and

$$
\Psi_{\epsilon}(z) \gtrsim \min \left\{\epsilon^{-1},\left|z_{1}\right|^{-1}\right\} \cdots \min \left\{\epsilon^{-1},\left|z_{n}\right|^{-1}\right\} \text {. }
$$

Using these estimates in place of (26) and (27), one can proceed as in the proofs of Lemma 2.4 and Theorem 1.2 and obtain the desired conclusion for $f \in \mathcal{S}\left(\mathbb{R}^{n}\right)$. 
Next, using the same argument as in the Step 2 of the previous proof, one infers (34).

The characterization (35) has the same proof as the corresponding formula (6).

(ii) The $M^{\infty}$ case uses the same argument as in the proofs of (7) and (8), with the group $U(2 n, \mathbb{R})$ replaced by $\mathbb{T}^{n}$.

\section{INTEGRAL REPRESENTATIONS FOR THE TORUS IN TERMS OF THE FACTIONAL FOURIER TRANSFORM}

Observe that the symplectic matrix in $U(2 n, \mathbb{R})$ corresponding to the complex matrix $S \in \mathbb{T}^{n}$ in (9) via the isomorphism $\iota$ in (16) is given by

$$
\iota(S)=\left(\begin{array}{cc}
A & -B \\
B & A
\end{array}\right)
$$

with

$$
A=\left(\begin{array}{ccc}
\cos \theta_{1} & & \\
& \ddots & \\
& & \cos \theta_{n}
\end{array}\right), \quad B=\left(\begin{array}{ccc}
\sin \theta_{1} & & \\
& \ddots & \\
& & \sin \theta_{n}
\end{array}\right) .
$$

Consider the case $\theta_{i} \neq k \pi, k \in \mathbb{Z}, i=1, \ldots, n$. The matrix $\iota(S)$ is a free symplectic matrix and the related metaplectic operator possesses the integral representation (14). Since

$$
A B^{-1}=B^{-1} A=\left(\begin{array}{lll}
\frac{\cos \theta_{1}}{\sin \theta_{1}} & & \\
& \ddots & \\
& & \frac{\cos \theta_{n}}{\sin \theta_{n}}
\end{array}\right),
$$

the polynomial $W\left(x, x^{\prime}\right)$ becomes

$$
W\left(x_{1}, \ldots, x_{n}, x_{1}^{\prime}, \ldots, x_{n}^{\prime}\right)=\sum_{i=1}^{n} \frac{1}{2 \sin \theta_{i}}\left(\cos \theta_{i} x_{i}^{2}-2 x_{i} x_{i}^{\prime}+\cos \theta_{i} x_{i}^{\prime 2}\right)
$$

and

$$
\Delta(W)=\frac{c}{\sqrt{\left|\sin \theta_{1} \cdots \sin \theta_{n}\right|}} .
$$

for some phase factor $c \in \mathbb{C}$, with $|c|=1$. Hence we obtain, for $\psi \in \mathcal{S}\left(\mathbb{R}^{n}\right)$,

$$
\widehat{\iota(S)} \psi(x)=\frac{c}{\sqrt{\left|\sin \theta_{1} \cdots \sin \theta_{n}\right|}} \int_{\mathbb{R}^{n}} e^{2 \pi i W\left(x, x^{\prime}\right)} \psi\left(x^{\prime}\right) d x^{\prime},
$$

with $W\left(x, x^{\prime}\right)$ in (30). From (31) we deduce that $\widehat{\iota(S)}$ can be written as the composition of the operators

$$
\widehat{\iota(S)}= \pm \widehat{\iota\left(S_{1}\right)} \cdots \widehat{\iota\left(S_{n}\right)}
$$


A CHARACTERIZATION OF MODULATION SPACES BY SYMPLECTIC ROTATIONS 15 where, for some phase factor $c$,

$$
\widehat{\iota\left(S_{i}\right)} \psi(x)=\frac{c}{\sqrt{\left|\sin \theta_{i}\right|}} \int_{\mathbb{R}} e^{\frac{\pi i}{\sin \theta_{i}}\left(\cos \theta_{i} x_{i}^{2}-2 x_{i} x_{i}^{\prime}+\cos \theta_{i} x_{i}^{\prime 2}\right)} \psi\left(x_{1}^{\prime}, \ldots, x_{i}^{\prime}, \ldots, x_{n}^{\prime}\right) d x_{i}^{\prime} .
$$

Indeed if $\theta_{i}=\pi / 2$, then $\widehat{\iota\left(S_{i}\right)}= \pm \widehat{J}$ is the Fourier transform with respect to the variable $x_{i}$. Otherwise, $\widehat{\iota\left(S_{i}\right)}= \pm \mathcal{F}_{\theta_{i}}$, the $\theta_{i}$-angle partial fractional Fourier transform (again referred to the variable $x_{i}$ ).

Alternatively, the same conclusion (32) can be drawn by writing

$$
S=\left(\begin{array}{ccc}
e^{i \theta_{1}} & & \\
& \ddots & \\
& & e^{i \theta_{n}}
\end{array}\right)=\left(\begin{array}{cccc}
e^{i \theta_{1}} & & & \\
& 1 & & \\
& & \ddots & \\
& & & 1
\end{array}\right) \cdots\left(\begin{array}{cccc}
1 & & & \\
& \ddots & & \\
& & 1 & \\
& & & e^{i \theta_{n}}
\end{array}\right)
$$

that is

$$
S=S_{1} \cdots S_{i} \cdots S_{n}
$$

with

$$
S_{i}=\left(\begin{array}{ccccccc}
1 & & & & & & \\
& \ddots & & & & & \\
& & 1 & & & & \\
& & & e^{i \theta_{i}} & & & \\
& & & & 1 & & \\
& & & & & \ddots & \\
& & & & & & 1
\end{array}\right), \quad i=1, \ldots, n
$$

so that

$$
\widehat{\iota(S)}=\iota\left(S_{1} \widehat{\ldots \iota \iota}\left(S_{1}\right)= \pm \widehat{\iota\left(S_{1}\right)} \cdots \widehat{\iota\left(S_{n}\right)} .\right.
$$

If $\theta_{i}=2 k \pi$ for some $k \in \mathbb{Z}, \widehat{\iota\left(S_{i}\right)}= \pm I$ with $I$ the identity operator. If $\theta_{i}=$ $(2 k+1) \pi$ for some $k \in \mathbb{Z}, \widehat{\iota\left(S_{i}\right)} \psi(x)= \pm \psi\left(x_{1}, \ldots,-x_{i}, \ldots, x_{n}\right)$.

Hence using the $\theta_{i}$-angle partial fractional Fourier transform $\mathcal{F}_{\theta_{i}}= \pm \widehat{\iota\left(S_{i}\right)}$ we can rephrase Theorem 1.3 as follows.

Theorem 4.1. Let $\varphi$ be the Gaussian of Theorem 1.2.

(i) For $1 \leq p<\infty, f \in M^{p}\left(\mathbb{R}^{n}\right)$, we have

$$
\|f\|_{M^{p}\left(\mathbb{R}^{n}\right)} \asymp\left(\int_{\mathbb{R}^{n} \times \mathbb{T}^{n}}\left|x_{1} \ldots x_{n} \|\left\langle f, \mathcal{F}_{\theta_{1}} \ldots \mathcal{F}_{\theta_{n}} T_{x} \varphi\right\rangle\right|^{p} d x d \theta_{1} \ldots d \theta_{n}\right)^{\frac{1}{p}},
$$

or, similarly,

$$
\|f\|_{M^{p}\left(\mathbb{R}^{n}\right)} \asymp\left(\int_{\mathbb{R}^{n} \times \mathbb{T}^{n}}\left|\xi_{1} \ldots \xi_{n} \|\left\langle f, \mathcal{F}_{\theta_{1}} \ldots \mathcal{F}_{\theta_{n}} M_{\xi} \varphi\right\rangle\right|^{p} d \xi d \theta_{1} \ldots d \theta_{n}\right)^{\frac{1}{p}} .
$$


(ii) For $p=\infty$,

or

$$
\|f\|_{M^{\infty}\left(\mathbb{R}^{n}\right)} \asymp \sup _{S \in \mathbb{T}^{n}} \sup _{x \in \mathbb{R}^{n}}\left|\left\langle f, \mathcal{F}_{\theta_{1}} \ldots \mathcal{F}_{\theta_{n}} T_{x} \varphi\right\rangle\right|
$$

$$
\|f\|_{M^{\infty}\left(\mathbb{R}^{n}\right)} \asymp \sup _{S \in \mathbb{T}^{n}} \sup _{\xi \in \mathbb{R}^{n}}\left|\left\langle f, \mathcal{F}_{\theta_{1}} \ldots \mathcal{F}_{\theta_{n}} M_{\xi} \varphi\right\rangle\right| .
$$

This concludes our study.

For sake of completeness, let us recall that integral representations involving metaplectic operators that do not arise from free symplectic matrices were studied in [14, 22].

\section{ACKNOWLEDGMENT}

The first and the third author have been supported by the Gruppo Nazionale per l'Analisi Matematica, la Probabilità e le loro Applicazioni (GNAMPA) of the Istituto Nazionale di Alta Matematica (INdAM). MdG has been financed by the Austrian Research Foundation FWF grant P27773.

\section{REFERENCES}

[1] A. Bényi, K. Gröchenig, K.A. Okoudjou and L.G. Rogers. Unimodular Fourier multipliers for modulation spaces. J. Funct. Anal., 246(2): 366-384, 2007.

[2] A. Bényi and K.A. Okoudjou. Time-frequency estimates for pseudodifferential operators. Contemporary Math., Amer. Math. Soc., 428:13-22, 2007.

[3] A. Bényi and K.A. Okoudjou. Local well-posedness of nonlinear dispersive equations on modulation spaces. Bull. Lond. Math. Soc., 41(3):549-558, 2009.

[4] A. Bényi, O. Tadahiro and O. Pocovnicu. On the probabilistic Cauchy theory of the cubic nonlinear Schrödinger equation on $\mathbb{R}^{d}, d \geq 3$. Trans. Amer. Math. Soc. Ser. B, 2:1-50, 2015.

[5] A. Cauli, F. Nicola and A. Tabacco. Strichartz estimates for the metaplectic representation. Submitted, 2017. arXiv:1706.03615.

[6] O. Christensen, H.G. Feichtinger and S. Paukner. Gabor analysis for imaging. Handbook of mathematical methods in imaging. Vol. 1, 2, 3, 1717-1757, Springer, New York, 2015.

[7] F. Concetti and J. Toft. Trace ideals for Fourier integral operators with non-smooth symbols, "Pseudo-Differential Operators: Partial Differential Equations and Time-Frequency Analysis", Fields Inst. Commun., Amer. Math. Soc., 52:255-264, 2007.

[8] F. Concetti, G. Garello and J. Toft. Trace ideals for Fourier integral operators with nonsmooth symbols II. Osaka J. Math., 47(3):739-786, 2010.

[9] E. Cordero, K. Gröchenig, F. Nicola and L. Rodino. Wiener algebras of Fourier integral operators. J. Math. Pures Appl., 99:219-233, 2013.

[10] E. Cordero and F. Nicola. Boundedness of Schrödinger type propagators on modulation spaces. J. Fourier Anal. Appl. 16(3):311-339, 2010.

[11] E. Cordero and F. Nicola. Schrödinger Equations with Bounded Perturbations. J. PseudoDiffer. Op. and Appl., 5(3):319-341, 2014.

[12] E. Cordero, K. Gröchenig, F. Nicola and L. Rodino. Generalized Metaplectic Operators and the Schrödinger Equation with a Potential in the Sjöstrand Class. J. Math. Phys., 55(8), art. no. 081506, 2014. 
[13] E. Cordero, F. Nicola and L. Rodino. Sparsity of Gabor representation of Schrödinger propagators. Appl. Comput. Harmon. Anal., 26(3):357-370, 2009.

[14] E. Cordero, F. Nicola and L. Rodino. Integral Representations for the Class of Generalized Metaplectic Operators. J. Fourier Anal. Appl., 21:694-714, 2015.

[15] M. A. de Gosson. Symplectic methods in harmonic analysis and in mathematical physics, volume 7 of Pseudo-Differential Operators. Theory and Applications. Birkhäuser/Springer Basel AG, Basel, 2011.

[16] H. G. Feichtinger. Modulation spaces on locally compact abelian groups, Technical Report, University Vienna, 1983, and also in Wavelets and Their Applications, M. Krishna, R. Radha, S. Thangavelu, editors, Allied Publishers, 99-140, 2003.

[17] H. G. Feichtinger. Choosing function spaces in harmonic analysis, Excursions in harmonic analysis. Vol. 4, Appl. Numer. Harmon. Anal., 65-101, Birkhäuser/Springer, Cham, 2015.

[18] H. G. Feichtinger. Modulation spaces: looking back and ahead. Sampl. Theory Signal Image Process., 5(2):109-140, 2006

[19] G. B. Folland. Harmonic analysis in phase space. Princeton Univ. Press, Princeton, NJ, 1989.

[20] K. Gröchenig. Foundations of time-frequency analysis. Applied and Numerical Harmonic Analysis. Birkhäuser Boston, Inc., Boston, MA, 2001.

[21] Leray, Jean Lagrangian analysis and quantum mechanics. A mathematical structure related to asymptotic expansions and the Maslov index, translated from the French by Carolyn Schroeder, MIT Press, Cambridge, Mass.-London, (1981).

[22] H. Morsche and P.J. Oonincx. Integral representations of affine transformations in phase space with an application to energy localization problems. CWI report, Amsterdam, 1999.

[23] M. Sugimoto and N. Tomita. Boundedness properties of pseudo-differential operators and Calderòn-Zygmund operators on modulation spaces. J. Fourier Anal. Appl., 14(1):124$143,2008$.

[24] M. Ruzhansky, B. Wang and H. Zhang. Global well-posedness and scattering for the fourth order nonlinear Schrödinger equations with small data in modulation and Sobolev spaces, J. Math. Pures Appl. (9),105(1):31-65, 2016.

[25] M. Ruzhansky, M. Sugimoto and B. Wang. Modulation spaces and nonlinear evolution equations, Evolution equations of hyperbolic and Schrödinger type, Progr. Math., 301:267283, Birkhäuser/Springer Basel AG, Basel, 2012.

[26] J. Toft. Continuity properties for modulation spaces, with applications to pseudodifferential calculus. I. J. Funct. Anal., 207(2):399-429, 2004.

[27] J. Toft. Continuity properties for modulation spaces, with applications to pseudodifferential calculus. II. Ann. Global Anal. Geom., 26(1):73-106, 2004.

[28] B. Wang, Z. Huo, C. Hao and Z. Guo. Harmonic analysis method for nonlinear evolution equations. I, World Scientific Publishing Co. Pte. Ltd., Hackensack, NJ, 2011.

[29] B. Wang, Z. Lifeng and G. Boling. Isometric decomposition operators, function spaces $E_{p, q}^{\lambda}$ and applications to nonlinear evolution equations. J. Funct. Anal., 233(1):1-39, 2006.

[30] B. Wang and H. Hudzik. The global Cauchy problem for the NLS and NLKG with small rough data. J. Differential Equations, 232(1):36-73, 2007. 
Dipartimento di Matematica, Università di Torino, Dipartimento di MatematiCA, Via CARlo Alberto 10, 10123 Torino, Italy

E-mail address: elena.cordero@unito.it

University of Vienna, Faculty of Mathematics, Oskar-Morgenstern-Platz 1 A-1090 Wien, Austria

E-mail address: maurice.de.gosson@univie.ac.at

Dipartimento di Scienze Matematiche, Politecnico di Torino, corso Duca Degli Abruzzi 24, 10129 Torino, Italy

E-mail address: fabio.nicola@polito.it 